J. Genet, Vol. 66, No. 1, April 1987, pp. 69-84. (C) Printed in India.

\title{
Evolution of cooperation by reciprocation within structured demes
}

\author{
N V JOSHI . \\ Centre for Theoretical Studies, Indian Institute of Science, Bangalore 560012 , India
}

MS received 2 February 1987

\begin{abstract}
The iterative two-person Prisoners' Dilemma game has been generalised to the $N$-person case. The evolution of cooperation is explored by matching the Tit For Tat (TFT) strategy (Axelrod and Hamilton 1981) against the selfish strategy. Extension of TFT to $\mathrm{N}$-person situations yields a graded set of strategies from the softest TFT, which continues cooperation even if only one of the opponents reciprocates it, to the hardest, which would do so only when all the remaining opponents cooperate.

The hardest TFr can go to fixation against the selfish strategy provided it crosses a threshold frequency $p_{c}$. All the other TFT are invadable by the selfish $(D)$ or the pure defector strategy, while none can invade $D$. Yet, provided a threshold $p_{c}$ is crossed, they can coexist stably with $D$. As $N$, the size of the group increases, the threshold $p_{c}$ also increases, indicating that the evolution of cooperation is more difficult for larger groups. Under certain conditions, only the soft TFT can coexist stably against the selfish strategy $D$, while the harder ones cannot. An interesting possibility of a complete takeover of the selfish population by successive invasions by harder and harder TFT strategies is also presented.
\end{abstract}

Keywords. Evolution of cooperation; group selection; reciprocation; game theory ; Tit For Tat.

\section{Introduction}

Explaining the evolution of cooperation within the framework of natural selection has been one of the challenging problems of evolutionary theory. If two individuals cooperate, each of them ought to be better off than otherwise. However, if one of them 'cheats', i.e., manages to get the benefit from the other without cooperating in turn, he is likely to be much better off. The cheaters are thus expected to be at a selective advantage. Trivers (1971) pointed out that this phenomenon may be modelled by the two-person Prisoner's Dilemma game. Given a choice between cooperation and cheating, it is always better to cheat since the cheater does better than the cooperator regardless of what his opponent chooses. Both the players thus opt for cheating and are consequently worse off than they would have been had they cooperated. Trivers also showed that if the same individuals interact repeatedly (iterated version of the Prisoner's Dilemma game) and can base their choices on the 'experience' gained in the previous encounters, cheating may not be the best strategy. He went on to show that a large degree of asymmetry between the benefits and costs associated with the acts of cooperation could lead to the evolution of reciprocal altruism.

A formal model for the evolution of cooperation based on reciprocal interactions has been investigated by Axelrod and Hamilton (1981). They consider the iterated version of the Prisoner's Dilemma game, and adopt a probabilistic treatment for the frequency of repeated interactions between the same pair of individuals. Under this scenario a rich variety of complex strategies is possible. From an analysis of 
these strategies they have been able to identify a set of robust and evolutionarily stable cooperative strategies.

In nature, however, an individual often interacts simultaneously with several individuals. It is therefore of interest to model such situations as $N$-person versions of the iterated two-person game. We therefore explore in this paper the outcomes of competition between the selfish strategy and the Tit For "Tat (TFT) strategy, proposed by A Rapoport, which was highly successful in the computer tournament organised by Axelrod (1984). An extension of TFT to $N$-person situations leads to a set of graded strategies, and we find that some of these can coexist in stable equilibria with the selfish strategy.

The basic model including the payoff matrices and the dynamics of genotype frequencies is described in $\$ 2$. The results of competition between two interacting strategies are described in $\$ 3$, while $\$ 4$ deals with situations involving more than two strategies at a time.

\section{The model}

\subsection{Payoffs to the players}

The two actions open to any player in a given game are to cooperate $(C)$ or defect $(D)$. For a two-person game, the four possible combinations of the choices by the two players are $C C, C D, D C$ and $D D$ where the successive letters indicate the choice made by the first and the second player, respectively. The same notation can be used to denote the payoff obtained by the first player as a result of the choices. Thus, if the outcome is $C D$, the payoff to the first player is also denoted by $C D$, and the value of the payoff to the second player $D C$. We assume that the elements of the payoff matrix satisfy the inequality $D C>C C>D D>C D$ as in the Prisoner's Dilemma.

In an $N$-person game, each participant faces $N-1$ opponents. Each can choose either $C$ or $D$. The payoff to any player then depends on his strategy, as well as the strategies chosen by his $N-1$ opponents. Let $n$ of the opponents of a player choose $C$ and let $N-1-n$ choose $D$. We take the payoff to a player choosing $C$ or $D$ as $f(C)$ or $f(D)$, where

$$
\begin{aligned}
& f(C)=[n \cdot C C+(N-1-n) \cdot C D] /(N-1), \\
& f(D)=[n \cdot D C+(N-1-n) \cdot D D] /(N-1) .
\end{aligned}
$$

In other words, we assume that the payoff accruing to a player is the average payoff the player would have received in a series of two-person games played with each of the opponents separately, the choices of strategies by each player remaining unaltered.

\subsection{The iterated Prisoner's Dilemma game}

In the interated version, the same participants may interact more than once. Under these conditions, the strategy for each game (interaction) could be specified in terms of the choices made by each player and his opponents in their previous interaction. The situations when players interact $M$ times is customarily referred to as a game consisting of $M$ moves. 
It is assumed that the game played by each group consists of at least one move. In one version, each game consists of exactly $M$ moves. Alternatively, the number of moves in the game played by each group could be a random variable. Following Axelrod and Hamilton (1981), the probability of continuation of the game is taken to be constant, denoted by $w$. Then the probability that the game consists of exactly $K$ moves is given by

$$
P(K)=w^{K-1}(1-w),
$$

and $M$, the mean number of moves in a game is $1 / w$.

\subsection{Dynamics of genotype frequencies}

Maynard Smith (1974) initiated the game theoretic approach to modelling competition between organisms; others have combined this with population genetics by equating payoffs with genetic fitnesses (Gadgil et al 1980; Hines 1980; Maynard Smith 1982). A similar approach is used here.

Consider an infinite population of asexual organisms with nonoverlapping generations, and with frequency $p$ and $1-p$ of genotypes $A$ and $B$, respectively. At the beginning of each generation, the population is subdivided into groups, with $N$ individuals per group. Assuming random association, the frequency of groups containing exactly $n$ individuals of genotype $A$ is given by

$$
F(N, n)=\left(\begin{array}{c}
N \\
n
\end{array}\right) p^{n}(1-p)^{N-n},
$$

where

$$
\left(\begin{array}{l}
N \\
n
\end{array}\right)=\frac{N !}{(N-n) ! n !}
$$

Each genotype is assumed to code for a specific strategy. The payoff of an individual of genotype $A$, when it is in a group containing $n$ opponents of type $A$, and when $K$ moves are made, is denoted by $Y_{A}(K, n)$.

The average payoff of $A$ is then given by

$$
f(A)=\sum_{n=0}^{N-1} F(N-1, n) \sum_{K=1}^{\infty} P(K) Y_{A}(K, n),
$$

where $P(K)$ is the probability that the $N$-tuple plays a game consisting of exactly $K$ moves.

We assume that the average payoff of $A$ measures the fitness of $A$. The mean fitness for the population is then seen to be

$$
f=p f(A)+(1-p) f(B),
$$

and the change in the frequency of $A$ from the $m$ th to $(m+1)$ st generation is given by

$$
p(m+1)=[f(A) / f] p(m) .
$$

All the offspring of a given generation mix and resettle in random associations of $N$ individuals each and then repeat the cycle to give rise to the next generation. 
Given the various payoffs associated with the strategies of the competing genotypes, one can thus study the changes in frequency of any genotype. This procedure can be readily generalised to a case where more than two genotypes interact.

\subsection{Elements of the payoff matrix}

As explained earlier ( $\$ 2.1)$, if an individual chooses the strategy $C$, his payoff for that game would be a linear combination of $C C$ and $C D$ (depending on the strategies of the rest of the competitors in the group), while a choice of $D$ would make it a linear combination of $D C$ and $D D$. Hence, the average payoff of a genotype would be a linear combination of $D C, C C, D D$ and $C D$. It can be seen that if $f(A)$ is greater than $f(B)$ for any value of frequency $p$, it will remain so if a constant term is added to each of the elements of the payoff matrix and/or if each is multiplied by a positive constant. Therefore, without loss of generality, we can take $D C=1, C C=c, D D=s$ and $C D=0$. Hence, the effects of variation in the values of the elements of the payoff matrix can be explored using only two parameters, viz., $c$ and $s$.

\subsection{The strategies}

We would like to explore the success of various cooperative strategies against the pure defector strategy. As mentioned earlier, in a two-person game, the Tit For Tat strategy of A Rapoport proved to be extremely successful in the 'tournaments' studied by Axelrod (1984). Hence, we consider here possible generalizations of the TFT strategy to $N$-person games. The TFT strategy uses information about the choices made by the opponent in the previous move (one-step memory). For mathematical convenience, we exclude strategies which can exploit other kinds of information (the number of moves remaining to be played, for example).

In an $N$-person game, each individual has $N-1$ opponents. We define a TFT strategy of the type $n(0 \leqslant n \leqslant N-1)$ as follows: cooperate on the first move. From the next move onwards, continue to cooperate if at least $n$ of the opponents have cooperated; if not, defect in the next move. Thus, $n=0$ corresponds to the pure cooperative strategy. In the two-person game, $n$ can be either 0 or 1 , with $n=1$ corresponding to the TFT strategy of Rapoport. In general, for an $N$ - person game, there would be $N-1$ TFT strategies. The strategy with $n=N-1$ is the hardest and $n=1$ the softest TFT. For other variants of TFT strategies, see Taylor (1975).

\section{Competition between two interacting strategies}

We consider first the competition between pure $D$ and pure $C$ strategists followed by competition between pure $D$ and various TFT strategies. It will be seen that whether one considers the total number of moves in a game as fixed (at $M$ ), or whether they follow the distribution described by (2) of $\$ 2.2$, the outcome is unchanged for a two-strategy interaction. However, these two lead to different outcomes when more than two strategies interact. This result, as well as some implications of assuming a fixed number of moves, would be discussed in $\$ 4$. 


\subsection{Competition between pure $D$ and pure $C$}

Let the frequency of pure $C$ in the population be $p$. The probability of a pure $C$ finding itself in a group containing exactly $n, C$ strategists amongst its opponents is $F(N-1, n)$, according to (3). With such a composition, payoff to $C$

$$
\begin{aligned}
& =[n \cdot c+(N-1-n) \cdot 0] /(N-1) \\
& =[n \cdot c /(N-1)] .
\end{aligned}
$$

If the games played by such an $N$-tuple consists of $K$ moves, the payoff is

$$
Y_{C}(K, n)=K . n . c /(N-1) \text {. }
$$

Hence, the average payoff of $C$ in the population is

$$
\begin{aligned}
f(C) & =\sum_{n=0}^{N-1} F(N-1, n) \sum_{K=0}^{\infty} P(K) . Y_{C}(K, n) \\
& =\sum_{n=0}^{N-1}\left(\begin{array}{c}
N-1 \\
n
\end{array}\right) p^{n}(1-p)^{N-1-n} \sum_{K=0}^{\infty} w^{K-1} \cdot(1-w) \frac{K . n \cdot c}{N-1},
\end{aligned}
$$

from (4), (2), (3) and (7). Regrouping the terms

$$
\begin{aligned}
f(C) & =\frac{c}{N-1} \sum_{n=0}^{N-1} n\left(\begin{array}{c}
N-1 \\
n
\end{array}\right) p^{n}(1-p)^{N-1-n}\left[\sum_{K=1}^{\infty} K w^{K-1}(1-w)\right] \\
& =\frac{c}{N-1}(N-1) p \cdot M \\
& =c p M
\end{aligned}
$$

The term in the square bracket is the average number of moves in the games played by an $N$-tuple. The payoff is thus seen to depend only on the mean value and not on the distribution of the number of moves in the games played.

A very similar calculation shows that for $D$,

$$
\begin{aligned}
Y_{D}(K, n) & =K[1 . n+(N-1-n) \cdot s] /(N-1) \\
& =K s+K .(1-s) /(N-1)
\end{aligned}
$$

and

$$
f(D)=p M(1-s)+M s
$$

Hence

$$
f(D)-f(C)=p \cdot M \cdot(1-c)+(1-p) \cdot M \cdot s .
$$

This will be $>0$ for all values of $p$, indicating that $D$ would continue to increase in the population for any value of $p$. Hence pure $D$ goes to fixation when in competition with pure $C$. 


\subsection{Competition between pure $D$ and hardest TFT}

The hardest TFT strategist requires all the opponents to cooperate for it to continue cooperation in the subsequent moves. Hence, in all the $N$-tuples where at least one $D$ is present, the TFT switch over to defection after cooperating in the first move. Only in the $N$-tuple with all TFT, $C$ is chosen in every move.

If the frequency of TFT is $p$, it is readily seen that

$$
\begin{aligned}
f(D)= & \sum_{n=0}^{N-1} F(N-1, n)\{[(1-w) \cdot(n \cdot 1+(N-1-n) \cdot s]\} /(N-1) \\
& +\sum_{k=2}^{\infty} K . P(K) \cdot s \\
= & p(1-s)+M . s .
\end{aligned}
$$

Interestingly, this is independent of $N$. For the TFT,

$$
\begin{aligned}
f(\mathrm{TFT})= & \sum_{n=0}^{N-2} F(N-1, n)\left[\frac{(1-w) \cdot n \cdot c}{N-1}+\sum_{K=2}^{\infty} K \cdot P(K) \cdot s\right] \\
& \quad+F(N-1, N-1) \sum_{K=2}^{\infty} K \cdot c \cdot P(K) \\
= & p^{N-1}(M-1)(c-s)+p \cdot c .+(M-1) \cdot s .
\end{aligned}
$$

Comparison of (12) and (13). shows that at $p=0, f(D)>f(\mathrm{TFT})$, i.e., $D$ is uninvadable by TFT. On the other hand, at $p=1$,

$$
f(D)-f(\text { TFT })=1+(M-1) \cdot s-M \cdot c .
$$

If $M, s$ and $c$ have values such that $f(D)<f(C)$ then TFT is also uninvadable by $D$. In fact, the line

$$
s=(M . c-1) /(M-1),
$$

separates the region in $(c, s)$ space where TFT is uninvadable (below the line) from the one where it is invadable (figure 1). With increasing $M$, a larger region in the $(c, d)$ space becomes favourable to TFT. As expected, the minimum value of $M$ needed for pure TFT to be uninvadable increases with $s$ and decreases with increasing $c$.

For the range of parameters where TFT is uninvadable, there is a critical value of $p$ given by

$$
p_{c}^{N-1}(M-1)(c-s)-p_{c}(1-c-s)-s=0,
$$

where the fitness of the competing strategies is equal. Since $f(D)$ increases linearly with $p, f(C)$ increases monotonically with $p, f(D)>f(C)$ at $p=0$ and $f(D)<f(C)$ at $p=1$, there is only one solution to this equation in the range $0=1$. Morcover, this equilibrium between the two strategies is unstable. In other words, once TFT crosses the threshold frequency $p_{c^{\prime}}$ (by various mechanisms, such as invasion by clusters, discussed by Axelrod and Hamilton 1981), it will go to fixation. 


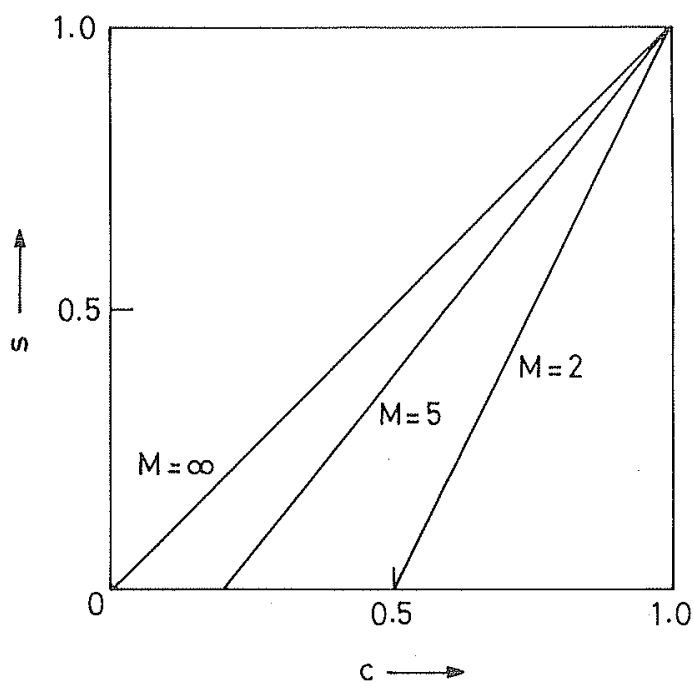

Figure 1. Regions in $(c, s)$ space where the Hardest TFT is uninvadable by $D$. For a given value of $M$, the mean number of moves in the game, the region favourable to TFT lies below the corresponding line.

It is seen from (15) that $p_{c}$ increases with $s$ and decreases with increasing $M$ and/or $c$ as expected. Other parameters remaining constant, $p_{c}$ increases with increasing $N$, suggesting that evolution of cooperation by this mechanism is more difficult in larger groups.

\subsection{Competition between pure $D$ and soft TFT}

3.3a General formulation: Consider a soft TFT strategy $S$ which continues to cooperate for the next move provided at least $m$ of its opponents cooperate during the current move. The payoff of such a strategy in an $N$-tuple with $n$ TFT strategists would be

$$
\begin{aligned}
Y_{S}(K, n) & =K \cdot n . c /(N-1), \quad n \geqslant m, \\
& =n \cdot c /(N-1)+(K-1) \cdot s, \quad n<m .
\end{aligned}
$$

The payoff of $D$ in a similar situation is

$$
\begin{aligned}
Y_{D}(K, n) & =K \cdot[n+(N-1-n) \cdot s] /(N-1), \quad n \geqslant m, \\
& =[n+(N-1-n) \cdot s] /(N-1)+(K-1) . s, \quad n<m .
\end{aligned}
$$

Using these equations, the expressions for the average payoffs of $D$ and $S$ can be written down explicitly. Both $f(S)$ and $f(D)$ are polynomials in $p$ (the frequency of $S$ in the population), with positive coefficients. The curves $f(S)$ and $f(D)$ increase monotonically with $p$ and are concave upwards (e.g. figure 2). When $p=0$, both $D$ and $S$ find themselves with all the $N-1$ opponents as $D$. The average payoff of $D$ in such a case is $M . s$, while that of $S$ is $(M-1) . s$ (since it cooperates in the first move); $D$ is thus uninvadable by $S$. Near $p=1$, each of them have $(N-1)$ opponents of the $S$ type who cooperate for all the games. Hence, 

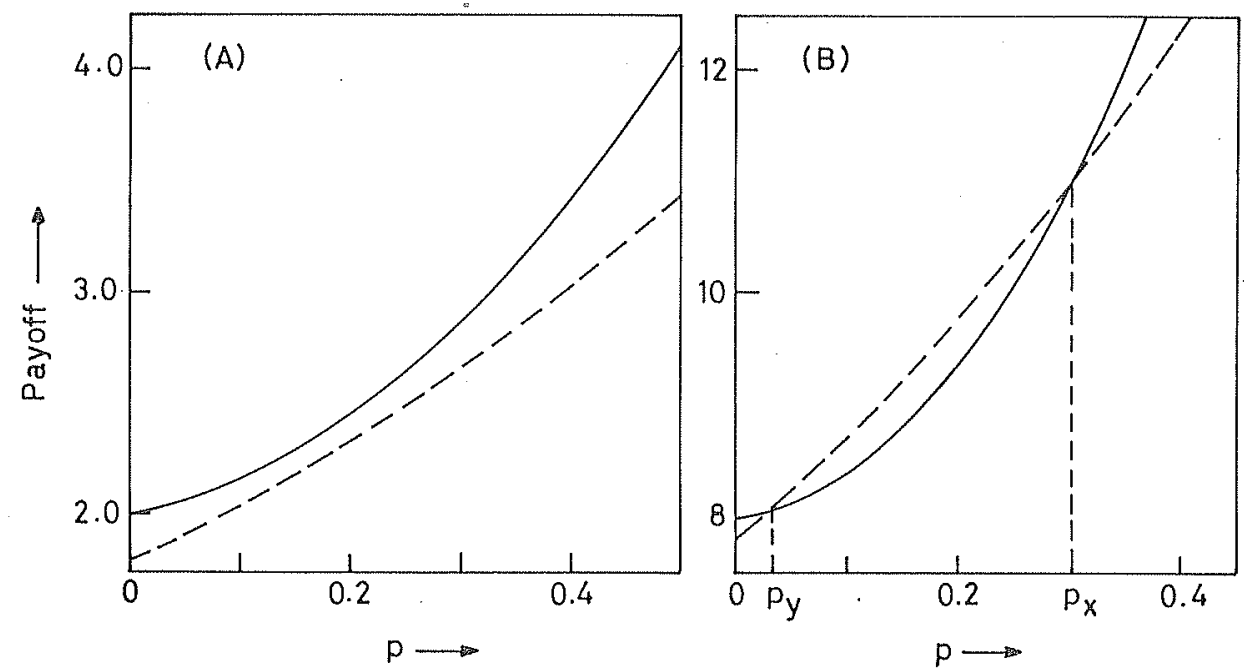

Figure 2. Payolfs to TFT (-- ) and $D(-)$ as functions of $p$, the frequency of Solt TFT for $N=3$, $c=0.6$ and $s=0.2$. A) For $M=10, D$ always goes to fixation. B) For $M=40$, a stable coexistence between TFT and $D$ is possible. The values of frequency $p_{y}$ and $p_{x}$ correspond to unstable and stable equilibrium, respectively.

$f(D)=M>f(S)=M . c$ and $S$ is invadable by $D$ (figure $2 \mathrm{~A}$ ). . Under these circamstances, $D$ is expected to go to fixation.

Interestingly, though $f(D)>f(S)$ at both $p=0$ and $p=1$, for some combination of the parameters $M, s$ and $c$, it is possible for the fitness of $S$ to be higher than that of $D$ for some intermediate values of $p$ (figure 2B). Under such circumstances, the curves $f(D)$ and $f(S)$ intersect at two points; at these values of $p$, the two strategies are in equilibrium. It is seen from figure $2 \mathrm{~B}$ that the point corresponding to $p_{v}$ is an unstable equilibrium, whereas the one corresponding to $p_{x}$ is a stable one. In other words, once the strategy $S$ is able to cross the threshold $p_{y}$, it would be able to coexist stably with $D$, at a frequency $p_{x}$.

3.3b Soft TFT for $N=3$ : For a three-person game, only one soft TFT is possible, viz., to continue cooperation even if only one of the other two opponents cooperates in the previous move. Using (16) and (17), and the procedure given in $\$ 3.3 \mathrm{a}$, the average payoffs as a function of the frequency $p$ of the TFT can be written as

$$
\begin{aligned}
& f(D)=p^{2}(M-1)(1-s)+p(1-s)+M s, \\
& f(S)=p^{2}(M-1) s+p[M c-2(M-1) s]+(M-1) s .
\end{aligned}
$$

The fitness of $D$ is greater than that of TFT at both $p=0$ and $p=1$. For a given value of $M$, one can determine the combination of $c$ and $s$ such that the two curves $f$ (TFT) and $f(D)$ touch at one point; the fitnesses of TFT and $D$ are equal at that point whereas at any other point, $f(D)$ is greater than $f(\mathrm{TFT})$. Any higher value of $c$ or lower value of $s$ then yields two values of $p\left(p_{y}\right.$ and $\left.p_{x}\right)$ where the fitnesses of the competing strategies become equal, and for $p_{y}<p<p_{x}$ we have $f$ (TFT) greater than $f(D)$. Hence it is possible for the two strategies to coexist. Regions in $(c, s)$ space 


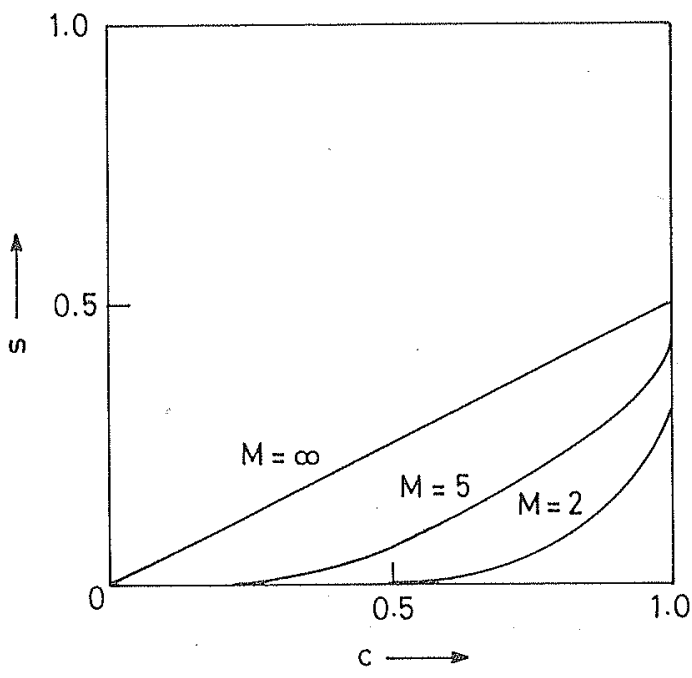

Figure 3. Regions in $(c, s)$ space where a Soft TFT $(N=3)$ can coexist with $D$. For a given value of $M$, the region favourable to TFT lies below the corresponding curve.

where such a coexistence between $S$ and $D$ is possible are shown in figure 3 for a few values of $M$.

As expected, the region where coexistence is possible increases with increasing $M$. Low values of $s$ and high values of $c$ favour coexistence. A comparison of figures 1 and 3 is of interest. It is seen from figure 1 that as $M \rightarrow \infty$, in the entire region $c>s$, the hard TFT is uninvadable by $D$. On the other hand, as seen from figure 3 , even as $M \rightarrow \infty$, the region favourable for a soft TFT is much smaller.

Is there, then, any advantage at all which a soft TFT enjoys over a hard TFT? Table 1 gives the values of threshold frequencies for various values of $M$ for typical values of $c$ and $s$. It is seen from the table that under some circumstances the threshold which a soft TFT needs to cross in order to establish itself in a stable coexistence with $D$ is lower than the one needed for the hard TFT. Hence, the former is more likely to establish itself in a population though it would never go to fixation.

Table 1. Some values of $s, c$ and $M$ where a Soft TFT has a lower threshold frequency compared to the Hardest TFT.

\begin{tabular}{rrrrrrr}
\hline$N$ & $c$ & $s$ & $M$ & Soft TFT & $\begin{array}{c}\text { Threshold } \\
\text { Soft TFT }\end{array}$ & $\begin{array}{c}\text { Threshold } \\
\text { Hardest TFT }\end{array}$ \\
\hline 3 & 0.85 & 0.25 & 5 & $S(2,1)$ & 0.250 & 0.302 \\
3 & 0.75 & 0.15 & 10 & $S(2,1)$ & 0.040 & 0.176 \\
4 & 0.95 & 0.15 & 5 & $S(3,1)$ & 0.089 & 0.331 \\
4 & 0.85 & 0.15 & 10 & $S(3,1)$ & 0.048 & 0.287 \\
4 & 0.85 & 0.25 & 5 & $S(3,2)$ & 0.297 & 0.441 \\
4 & 0.65 & 0.05 & 10 & $S(3,2)$ & 0.092 & 0.294 \\
5 & 0.65 & 0.05 & 5 & $S(4,1)$ & 0.048 & 0.546 \\
5 & 0.50 & 0.05 & 10 & $S(4,1)$ & 0.032 & 0.513 \\
5 & 0.95 & 0.15 & 5 & $S(4,2)$ & 0.158 & 0.427 \\
5 & 0.99 & 0.35 & 10 & $S(4,2)$ & 0.272 & 0.433 \\
5 & 0.75 & 0.25 & 5 & $S(4,3)$ & 0.500 & 0.594 \\
5 & 0.85 & 0.45 & 10 & $S(4,3)$ & 0.472 & 0.532 \\
\hline
\end{tabular}


3.3c Soft TFT for $N>4$ : Using the equations described in $\$ 3.3 a$. and the procedure outlined in $\$ 3.3 \mathrm{~b}$, one can obtain the regions of coexistence (allowed regions) in the $(c, s)$ space for various soft TFT strategies for any value of $N$. Qualitatively, the results are as expected. As seen from figure 4 , the allowed regions for the softest strategy for $N=4$ is very small, for the less soft it is slightly larger, and so on. The allowed regions for all these strategies increase with increasing $M$.

An interesting result seen from figure 4 is that a softer strategy may be able to coexist with $D$, while the harder one may be unable to do so. For example, for $N=4$, at $c=0.350, s=0.03$ and $M=10, S(3,1)$ can coexist with $D$, while for $S(3,2)$ no coexistence is possible.

The advantage of the harder strategy lies in it being less prone to exploitation by $D$, while the disadvantage is in denying cooperation to some members of its own kind during the process; whereas for the softer of the two strategies, the situation is exactly the reverse. Over a limited range of parameter values, the balance seems to tilt in favour of the softer strategy. The regions where this happens are very small, and are characterized by low values of $s$.

It is possible to define a soft TFT not just in terms of the minimum number of cooperating opponents but in terms of the proportion of the cooperating opponents required by it for continuing cooperation. For example one can define a soft TFT which continues cooperation if at least half of its opponents cooperate, and defects otherwise. It is seen, however, that the regions for such a strategy in $(c, s)$ space where it can coexist with $D$ are not independent of $N$, but decrease with increasing $N$. Thus, the TFT strategy $S(10,5)$ which continued cooperation when at least 5 of its 10 opponents cooperated had a larger allowed region compared to, say, $S(20$, 10).

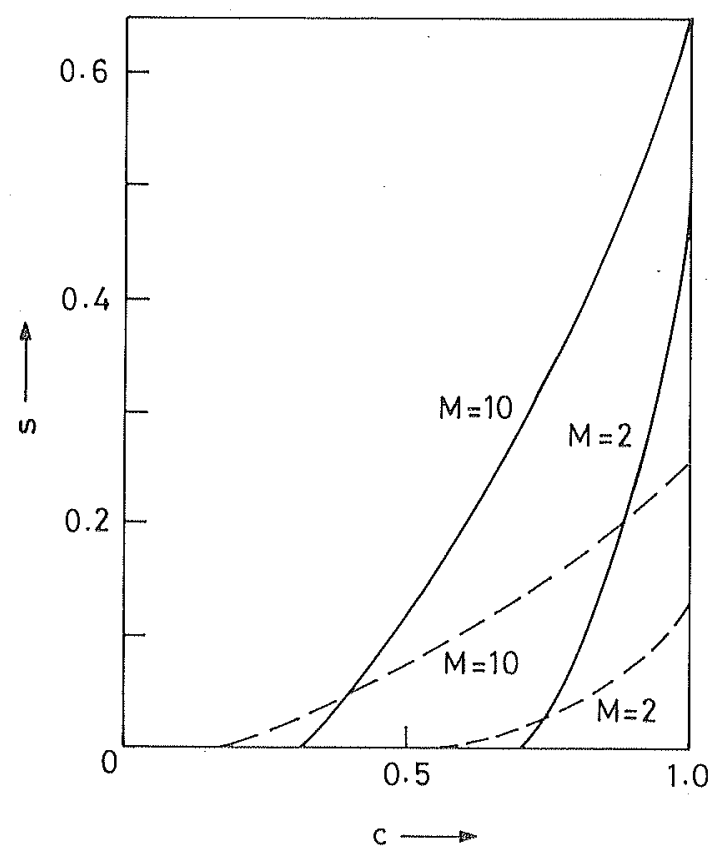

Figure 4. Regions in $(c, s)$ space where Solt TFT can coexist with $D$, for $N=4$, $M=2$ and also $M=10$. The region favourable to the Soltest TFT lies below the dashed curves, while that for the harder strategy, lies below the solid curve. 
To understand this result, we compare the payoff to a soft TFT $S(N, m)$ facing $N$ opponents $\left[n\right.$ soft TFT and $\left.(N-n) D^{\prime} s\right]$ with that to a $D$ in a similar situation. If $n<m$, we get

$$
Y_{S}(K, n)=n . c / N+(K-1) s<Y_{D}(K, n)=n .(1-s) / N+K . s,
$$

and if $n>m$, still

$$
Y_{S}(K, n)=K . n . c / N<Y_{D}(K, n)=[K . n .(1-s) / N]+K . s .
$$

However, when $n=m$,

while

$$
Y_{S}(K, m)=K . m \cdot c / N
$$

$$
Y_{D}(K, m)=[m(1-s) / N]+K . s .
$$

If the values of $K$ and $c$ are high enough, and that of $s$ low enough, it is possible for $Y_{S}(K, n)$ to be greater than $Y_{D}(K, n)$. Howwever, this is only a necessary condition. For $f(S)$ to be greater than $f(D)$, this advantage gained from the $N$-tuples with $n=m$ has to more than outweigh the disadvantage attained in the other $N$-tuples. For this to happen, $p$, the frequency of $S$, should be such that the frequency of $N$-tuples with $n=m$ is high, i.e., $F(N, m)$ should be maximized [(3)] with respect to $p$. It can be seen that the required value of $p$ is equal to $m / N$.

However, the maximum value of $F(N, n)$ decreases with increasing $N$. For $F(N, N / 2)$ for example, the maximum value of $F(6,3)$ is 0.3125 , of $F(8,4)$ is 0.2734 and $F(10,5)$ is $0 \cdot 2461$. Hence, the allowed regions for soft TFT shrink rapidly as $N$ increases, indicating once again that for large group sizes it is more difficult for cooperation to evolve.

\section{Competition involving more than two strategies}

The next logical step is to consider interactions where more than two strategies are involved. It is of interest to see whether the equilibrium between a soft TFT and $D$ is invadable by a harder TFT. The motivation for such an analysis is provided from the following results seen for $N=4$ for certain combinations of $c, s$ and $\mathrm{M}$ : all the soft strategies can coexist with $D$. The softest of them, $S(3,1)$ has the lowest threshold. However, its equilibrium frequency is higher than the threshold of the next harder strategy, $S(3,2)$. Its equilibrium frequency, in turn, is higher than the threshold frequency of the hardest TFT strategy, which has the potential for taking over the population completely. It is tempting to explore whether a cascade process $[S(3,1)$ invading $D, S(3,2)$ invading $S(3,1)-D$ and $S(3,3)$ invading $S(3,2)-D]$ can result in the complete elimination of $D$.

To start with, the simplest case $(N=3)$ is considered. The frequencies of the Soft TFT $(S)$ and Hard TFT $(H)$ are denoted by $p_{S}$ and $p_{H}$ respectively, and we investigate whether $H$ can invade the $S$ - $D$ equilibrium. For it to be able to do so, its payoff at this equilibrium should be higher than that of $S$ (or $D$ ). When invading an $S-D$ equilibrium, $H$ would face, in a triplet either $S S, D D$ or $S D$ as opponents. Now the payoff of $H$ and $S$ are identical against $S S$ and $D D$ opponents. However, payoff of $H$ against $S D$ is 


$$
\begin{aligned}
& =c / 2+P(2) \cdot(1+s) / 2+s \cdot \sum_{K=3}^{\infty} P(K) \cdot(K-2) \\
& =c / 2+[(1+s) \cdot(M-1) / 2]+\left[(M-1)^{2} / M\right] . s,
\end{aligned}
$$

while that of $S$ against $S D$ is simply $M . c / 2$.

Thus, for $H$ to successfully invade $S-D$ equilibrium, $c$ should be small and $s$ "should be large. These conditions, however, are exactly those which would render a stable equilibrium between $D$ and $S$ difficult.

The condition for equal fitness for $H$ and $S$ when $H$ invades an $S$ - $D$ equilibrium is described by (using the above values of payoffs and simplifying)

$$
s .(2 M-1)-M \cdot c+1=0 \text {. }
$$

As seen in figure 5 , this line cuts the $s=0$ line at $1 / M$, and $c=1$ line at $(M-1) /(2 M-1)$; and in the region above the line, invasion is possible. However, as seen from figure 3 [and as can be derived from (18)], these are exactly the points where the concave curve delineating the allowed region for $S$ cuts the two lines $s=0$ and $c=1 ; S$ can coexist with $D$ below this curve. Therefore, $H$ will never be able to invade the $S$ - $D$ equilibrium.

Consider now a situation where the total number of moves in the game is fixed at $M$. As Axelrod and Hamilton (1981) have shown, pure $D$ is an evolutionarily stable strategy (ESS) under such a situation. To arrive at this result they have invoked

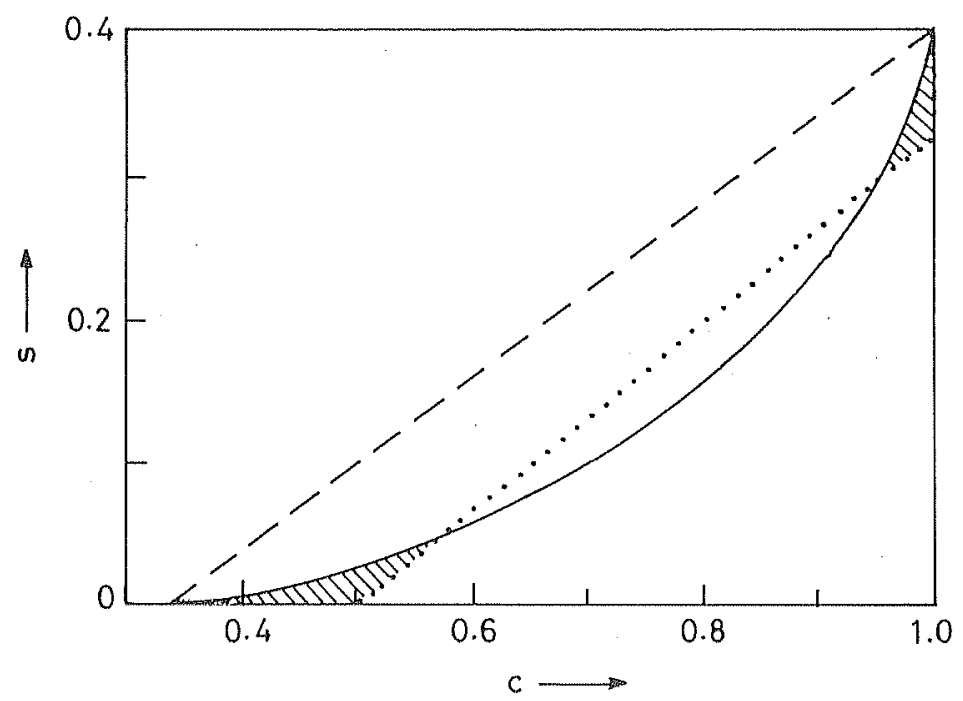

Figure 5. Invasion of the Soft TFT- $D$ equilibrium by the Hardest TFT for $\mathrm{N}=3, M=3$. A stable equilibrium between Soft TFT- $D$ is possible only in the region below the curve. Invasion by the hardest TFT is possible only in the region above the dashed line if the number of moves in the game is geometrically distributed with mean $M$, and above the dotted line if the number of moves in the game is fixed at $M$. Accordingly, such an invasion is impossible for the former, while it is possible in the hatched regions for the latter. 
strategies which involve a 'knowledge' of $M$, e.g., be a TFT upto $M-1$ moves and $D$ at the last move, TFT upto $M-2$ moves and $D$ for the last two moves etc. We, however, assume that the players have no 'knowledge' of $M$, and a strategy is defined only in terms of the choices made by the player and his opponents upto that move.

Thus when the games played by each triplet consists of exactly $M$ moves, the payoff of $H$ against $S D$ becomes $c / 2+(1+s) / 2+(M-2)$. $s$, while that of $S$ against $S D$ remains $M c / 2$. Hence, the condition for $H$ to be able to invade $S-D$ becomes

$$
\text { s. }(2 M-3)-(M-1) \cdot c+1>0 \text {. }
$$

As seen from figure 5, there are two small regions, one near $s=0$ and the other near $c=1$, where a coexistence between $S$ and $D$ is possible, and this equilibrium is invadable by $H$.

When such a situation occurs, under certain values of $c, s$ and $M$, one can observe the following effect. The threshold needed for $H$ to be able to invade $D$ is higher than that needed by $S$. Therefore, $S$ has a better chance of crossing the threshold, and reaching a stable equilibrium with $D$. Now, $H$ can invade this combination and eventually eliminate $D$. The final composition of the population would be a mixture of $S$ and $H$, depending on the size of the initial perturbation by $H$. In such a population, $C, S$ and $H$ are indistinguishable. One has thus seen evolution of cooperation taking place successfully against a selfish $(D)$ strategy.

Analysis of an $N=4$ case also reveals similar behaviour. When the number of games is distributed according to (2), no harder strategy can invade an equilibrium between $D$ and the softer strategy. However, when the number of games is fixed, for some range of parameter values (a typical example being $M=2, c=0.85$ and $s=0.05)$, one can see the equilibrium between $D$ and the Softest $[s(3,1)]$ being invadable by the next harder strategy $S(3,2)$, and in turn, the $S(3,2)-D$ equilibrium being invadable by the Hardest strategy, resulting in the complete elimination of $D$.

Such a takeover of a selfish population by. successive invasions by gradually hardening TFT strategies may be of considerable interest. It must be emphasized, however, that the region in the parameter space where such behaviour is observed is extremely small.

\section{Discussion and conclusions}

Evolution of cooperation by reciprocation has been investigated in spatially structured populations. The choice of cooperation (or otherwise) by an individual is assumed to be influenced by the choices made by the other individuals in the group. Though 'strategy dependent selection' may be a better description for evolution occurring under this scenario, the framework of the $N$-person game theory is particularly suitable for analysing such situations (Charnov 1982; Maynard Smith 1982; Riechert and Hammerstein 1983). Gregariously living organisms exploiting a common resource-primate troops, breeding colonies of birds etc. - seem to be suitable systems for the applications of this model (Lombardo 1985).

We assume that the population is subdivided into groups containing $N$ individuals each. The average fitness of an individual in groups with a higher proportion of TFT tends to be higher. Hence, following Wilson $(1975,1980)$ the 
evolution of cooperation in the present model can be described as an example of group selection. The differences in the average fitnesses of different groups are caused, however, by the reciprocal interactions between individual strategies.

Brown et al (1982) have presented a detailed analysis of the evolution of cooperation by reciprocation, using the TFT strategy. They have shown that as the ratio $\alpha / \beta$ increases (where $\alpha$ is the total number of interactions which an individual experiences in a generation and $\beta$ is the number of interactions which are with individuals perceived as strangers), the threshold required by the TFT strategy for invading $D$ becomes smaller. They have very ingeniously obtained values of $\alpha$ and $\beta$ under a variety of conditions such as single partner/multiple partner models, finite/infinite memory, haploid/diploid organisms etc. and have also cast their model in a form such that their predictions can be compared to those made by Kin Selection theory. Another interesting and recent study of the evolution of cooperation (more specifically, helping behaviour) is by Peck and Feldman (1986). Using biologically plausible assumptions, they have shown that the threshold required by TFT for invading $D$ can be made arbitrarily small. They have also investigated simultaneous competition involving $C, D$, and TFT, and have reported an interesting situation where invasion by $D$ of a neutral equilibrium between $C$ and TFT is initially successful, but leads eventually to fixation of TFT. Both these studies, however, have specifically considered situtions when population structure is absent. The present model, on the other hand, explores the evolution of cooperation in a structured population.

For two competing strategies $A$ and $B$, if $A$ is able to invade $B$, and is, in turn, uninvadable by $B$, it is generally taken to mean that $A$ goes to fixation. We however show that where fitness has a nonlinear dependence on frequencies, it is still possible for the two strategies to coexist in stable equilibrium even if both the above conditions hold.

The present study has also shown that for a cooperative strategy to succeed against a selfish (defector) strategy, under certain conditions it is better to extend cooperation even though there is a risk of being exploited by a defector. On the other hand, under other conditions it is better to be more discriminating. We have also seen that a selfish population may be taken over by successive invasions by more and more discriminating strategies, though very discriminating strategy is unable to make any impact to start with against the selfish strategy.

It is of interest to examine the effects of relaxing some of the assumptions of the model presented in this paper. In diploid organisms with a one-locus-two-allele system, the two homozygotes TT and $D D$ may be identified with TFT and $D$ strategies. If $T$ is recessive and if $p$ is the frequency of $T$, then under Hardy-Wienberg equilibrium, the frequency of TFT strategists will be $p^{2}$. An examination of (15) indicates that the threshold frequency which a hard TFT has to cross in order to go to fixation is higher for diploids $\left(p_{c}{ }^{1 / 2}\right)$ compared to haploids $\left(p_{c}\right)$ for a recessive gene. A similar result holds for a soft TFT [(18)]; however, the equilibrium frequency (coexistence with $D$ ) is also higher. If $T$ is dominant, the thresholds are lower $\left[1-\left(1-p_{c}\right)^{1 / 2}\right]$, and the equilibrium frequency for a soft TFT is also lower. A further modification may be to consider the strategies as continuously varying traits coded for by a large number of genes. Aoki $(1983,1984)$ has analysed the evolution of TFT (and other) strategies in considerable detail, and has shown that biallelic models lead to predictions that are qualitatively and quantitatively 
different from those of polygenic models. A similar result is expected for the present model as well.

The assumption of random association in the formation of trait groups is also biologically unrealistic. Wilson (1980) has indicated that the variance between groups for most natural populations is more than that expected under a binomial distribution. Several mechanisms can lead to such an increase in the varianceassortative mating, kin recognition, high population viscosity etc. The net effect, however, is that the TFT are more likely to be associated with other TFT and the $D$ 's with $D$ 's. This would lower the threshold frequency for TFT's, indicating that the conditions for evolution of cooperation by reciprocation may be somewhat less stringent than predicted by the model. Finally, the assumption of infinite population size suppresses the effects of stochastic variations in the composition of groups. Studies with finite populations, which explore the effects of stochastic variations in group compositions and group sizes as well as in the elements of the payoff matrix are in progress.

The model considered here, albeit simple, has provided some insights into the different types of phenomena which may occur during evolution of cooperation. The Axelrod-Hamilton approach is being successfully used for analysing conflict situations in animals. Lombardo (1985) has described the mutual restraint in tree swallows as an example of the Tit For Tat strategy in an iterated Prisoner's Dilemma game. With more such studies forthcoming, it would be possible to verify the predictions of the present model.

\section{Acknowledgements}

It is a pleasure to thank Professors Madhav Gadgil and Sulochana Gadgil for introducing me to the subject, for many fruitful discussions, for encouragement and for numerous suggestions towards improvement of the manuscript. I also thank two anonymous reviewers for many helpful comments on an earlier version of the manuscript.

\section{References}

Aoki K 1983 A quantitative genetic model for reciprocal altruism: A condition for kin or group selection to prevail. Proc. Natl. Acad. Sci. USA 80: 4065-4068

Aoki K 1984 Quantitative genetic model of two policy games between relatives. J. Theor. Biol, 109: $111-126$

Axelrod R 1984 The evolution of cooperation (New York: Basic Boolss)

Axelrod R and Hamilton W D 1981 The evolution of cooperation. Science 211: 1390-1396

Brown J L, Sanderson M J and Michod R E 1982 Evolution of social behaviour by reciprocation. J. Theor. Biol, 99: 319-339

Charnov E L 1982 The theory of sex allocation (New Jersey: Princeton Univ. Press)

Gadgil S, Nanjundiah V and Gadgil M 1980 On evolutionarily stable compositions of populations of interacting genotypes. J. Theor. Biol. 84: 737-760

Hines W G S 1980 An evolutionarily stable strategy model for randomly mating diploid populations. J. Theor. Biol. 87: 507-513

Lombardo M P 1985 Mutual restraint in Tree Swallows: A test of the TIT for TAT Model of Reciprocity. Science 227: 1363-1365

Maynard Smith J 1974 The theory of games and the evolution of animal conflicts. J. Theor. Biol. 47: 209-221 
Maynard Smith I 1982 Evolution and the theory of games (New York: Cambridge Univ. Press)

Peck J R and Feldman M W 1986 The evolution of helping behaviour in large randomly mixed populations. Am. Nat. 127: 209-221

Riechert S E and Hammerstein P 1983 Game theory in the ecological context. Anru. Rev. Ecol. Syst. 14: $377-409$

Taylor M 1975 Anarchy and cooperation (London and New York: Wiley)

Trivers R L 1971 The evolution of reciprocal altruism. Q. Rev. Biol. - 46: 377-409

Wilson D S 1975 A theory of group selection. Proc. Nall, Acad. Sci. USA 72: 143-146

Wilson D S 1980 The natural selection of populations and communities (Menlo Park, CA: Benjamin-Cummings) 